\title{
Renin-angiotensin system mediated mechanisms: cardioreparation and cardioprotection
}

\author{
Christian G Brilla
}

The Framingham heart study has convincingly shown that, in the presence of left ventricular hypertrophy $(\mathrm{LVH})$ of any cause, there is an increased risk for all major cardiovascular events, including the development of cardiac failure. The risk of cardiac failure for men and women alike rises six to 18 times in the presence of LVH. ${ }^{1}$

\section{Left ventricular hypertrophy}

A number of changes occur within the myocardial structure during the development of $\mathrm{LVH}$. There is a tremendous increase in myocyte size in patients with dilated cardiomyopathy, hypertensive heart disease, coronary artery disease or post-myocardial infarction. The major physiological stimulus for myocytes to undergo hypertrophy is mechanical stress, or stretch, either provided by increased preload, or increased afterload, or a combined increase in both.

In a study looking at cultured cardiac myocytes under serum free conditions - that is, no hormones or growth factors were present-it was found that stretching caused myocytes to hypertrophy. ${ }^{2}$ There was upregulation of the protooncogenes c-fos and c-jun, and even more importantly, an overexpression of the contractile proteins myosin and actin. In addition to increased load, other triggers for myocyte hypertrophy include angiotensin II, catecholamines, thyroxin, and growth hormone.

In patients with chronic failure caused by hypertensive heart disease, endomyocardial biopsies show diffuse interstitial and perivascular fibrosis in addition to myocyte hypertrophy. This is true for the pressure overloaded left ventricle and the non-overloaded right ventricle.

COLLAGEN VOLUME FRACTION

When adverse changes within the cardiac interstitium are quantified using quantitative video densitometry to measure collagen volume fraction, an eightfold increase is found in patients with hypertensive heart disease. As type I collagen has a tensile strength which comes close to that of steel, any increase will unequivocally change the mechanical behaviour of the ventricle. ${ }^{3}$ There is also a significant increase, by $400 \%$, of collagen volume fraction in patients with dilated cardiomyopathy.

Khinikum der Philipps Universität, Zentrum f. Innere

Medizin/Kardiologie, Baldinger Strasse, 35043 Marburg, Germany C G Brilla

Correspondence to: Professor Brilla email: brilla@t-online.de ROLE OF ANGIOTENSIN II

The major stimulus for cardiac fibroblasts - the target cells that are responsible for the build up of collagen in the myocardium - is not load but angiotensin II. In a study measuring collagen synthesis in adult cardiac fibroblasts cultured under serum free conditions, angiotensin II induced a dose dependent significant increase in collagen synthesis counteracted by type I angiotensin II receptor antagonists such as losartan, and not by a type II receptor antagonist. ${ }^{4}$ Therefore, angiotensin II stimulates fibroblasts to increase collagen synthesis via the type I receptor.

In addition, angiotensin II is able to decrease the activity of matrix metalloproteinase-1 which is the key enzyme of interstitial collagen degradation. Here, the addition of a type II receptor antagonist completely blocks the angiotensin II mediated effect. ${ }^{4}$

In a study of rats with renovascular hypertension, stimulation of the circulating reninangiotensin-aldosterone system produced a significant increase in the density of mycocardial collagen fibres, which was progressive over time. ${ }^{5}$ The increase in collagen was associated with a progressive deterioration of cardiac function.

There are a number of stimuli for cardiac fibroblast growth and increased collagen synthesis, including angiotensin II and aldosterone. Other stimuli include transforming growth factor $\beta 1$ and insulin-like growth factor 1. Inhibitors of cardiac fibroblast growth include prostaglandin E2 and the cytokine interleukin 1 .

\section{Reversal of hypertrophy: animal studies}

To investigate if these adverse structural changes within the cardiac interstitium could be reversed, studies were performed with spontaneously hypertensive rats (SHRs) - the most analogous model for primary hypertension in man. LVH was examined at the beginning of the trial, at 14 weeks of age, and after three months treatment with the ACE inhibitor lisinopril. ${ }^{3}$

Two different dosage regimens of lisinopril were used. With a low dosage regimen, which inhibits the circulating renin-angiotensinaldosterone system in renovascular hypertension but does reduce elevated blood pressure in SHR, LVH did not regress. When a higher dosage of lisinopril was used sufficient to normalise blood pressure, there was complete regression of LVH.

There was significant fibrosis in untreated SHRs compared to age and sex matched controls. With either dose of lisinopril, even when blood pressure was not normalised and LVH not affected, fibrosis was completely
Interestingly, myocardial angiotensin II concentrations were clearly raised in untreated SHRs compared with controls. Either lisinopril dose was able to normalise local myocardial angiotensin II concentrations. 
Associated with these beneficial effects on myocardial structure, beneficial effects on myocardial diastolic stiffness were found. With high dose lisinopril, which normalised blood pressure and regressed LVH and fibrosis, there was normalisation of diastolic stiffness. The same was true when low dose lisinopril was used which did not regress $\mathrm{LVH}$, so that there was still myocyte hypertrophy and blood pressure was elevated as in untreated SHRs, but fibrosis was regressed and diastolic stiffness was normalised.

ADVANCED DISEASE

In another trial focusing on SHR but with advanced hypertensive heart disease, there was significant fibrosis compared with controls. Even in this advanced model of hypertensive heart disease, fibrosis could be significantly regressed, although not normalised, compared to untreated SHRs.

More importantly, at the end of the study, in untreated SHRs there was a significant drop in systolic function measured by myocardial contractility. With lisinopril treatment, this drop in systolic function of myocardial contractility was abolished.

\section{First clinical study}

Is it possible to regress fibrosis in man, and improve myocardial function? This question was addressed for the first time in a clinical trial presented at the 1999 American Heart Association meeting in Atlanta. ${ }^{7}$

STUDY DESIGN

This was a single centre, randomised, prospective, double blind parallel group trial, comparing lisinopril with the diuretic agent hydrochlorothiazide (HCTZ), in patients with primary arterial hypertension. The primary end point was myocardial fibrosis and the follow up period was six months.

The inclusion criteria were symptomatic primary arterial hypertension, with no coronary artery disease, excluded by coronary angiography, and no other cardiovascular disorder or systemic disease. The age range was $18-70$ years, with all patients having LVH measured by the Devereux criteria. All patients had signs of left ventricular diastolic dysfunction, measured by a Doppler E/A ratio less than one. Left ventricular endomyocardial biopsies were performed at the beginning and the end of the observation period to obtain hard data on myocardial structure.

RESULTS

Looking at collagen volume fraction it was found that under randomised, double blind conditions fibrosis was significantly regressed in patients treated with 5-20 mg lisinopril per day titrated to normalise blood pressure. In addition to collagen volume fraction, which was measured morphometrically, hydroxyproline concentrations of endomyocardial biopsy samples of the left ventricle were measured by high pressure liquid chromatography. It was found that there was a significant reduction of myocardial hydroxyproline concentration in lisinopril treated patients, which means a

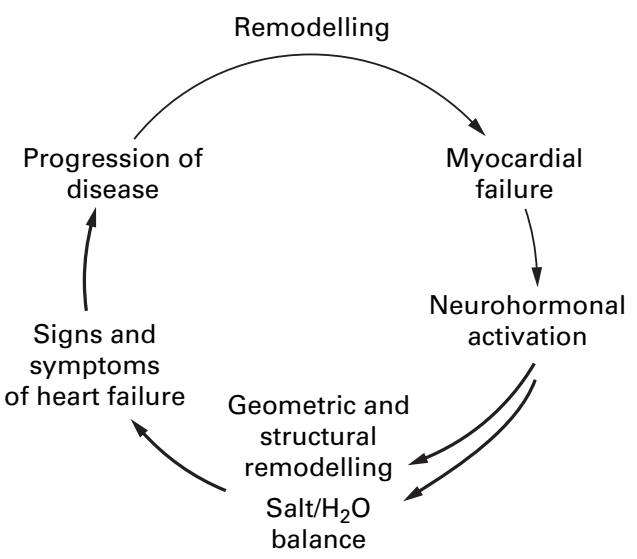

Figure 1 ACE inhibition is able to interrupt the vicious cycle of structural heart disease.

significant regression of fibrosis over just six months of treatment. There was no significant change of fibrosis in the control diuretic group (25-50 mg HCTZ per day), although blood pressure was controlled equally as well as in lisinopril treated patients.

Associated with these beneficial structural changes during lisinopril treatment was a significant improvement in the transmitral Doppler in-flow pattern, with a significant increase in the E/A ratio, showing that left ventricular diastolic function can be improved by regressing myocardial fibrosis. No significant change occurred in the HCTZ control group.

\section{Conclusion}

With myocardial failure due to any causehypertensive heart disease, primary dilated cardiomyopathy, or myocardial infarctionthere is upregulation and stimulation of the neurohormonal systems. This stimulation, particularly of the renin-angiotensin-aldosterone system, has detrimental effects on myocardial structure, with remodelling of myocyte and interstitial compartments of the myocardium, which further impairs myocardial function. This in turn activates the neurohormonal system, leading to the development of a vicious cycle. This vicious cycle can be effectively blocked by the use of ACE inhibitors (fig 1), and the occurrence of pathologic myocardial structure can be either prevented (cardioprotection) or reversed (cardioreparation).

\footnotetext{
Kannel WB, Ho K, Thom T. Changing epidemiological features of cardiac failure. Br Heart f 1987;28:269-75.

2 tures of cardiac failure. Br Heart f 1987;28:269-75. mycotes stimulates protooncogene expression. 7 Biol Chem mycotes stimulates

3 Brilla CG, Janicki JS, Weber KT. Impaired diastolic function and coronary reserve in genetic hypertension. Role of interstitial fibrosis and medial thickening of intramycocardial coronary arteries. Circ Res 1991;69:107-15.

4 Brilla CG, Zhou G, Matsubara L, et al. Collagen metabolism in cultured adult rat cardiac fibroblasts: response to angiotensin II and aldosterone. $\mathcal{F} \mathrm{Mol} \mathrm{Cell} \mathrm{Car-}$ diol 1994;26:809-20.

5 Doering CW, Jalil JE, Janicki JS, et al Collagen network remodeling and diastolic stiffness of the rat left ventricle with pressure overload hypertrophy. Cardiovasc Res 1988; 22:686-95.

6 Brilla CG, Matsubara L, Weber KT. Advanced hypertensive heart disease in spontaneously hypertensive rats. Lisinopril-mediated regression of myocardial fibrosis. Hinpertension 1996;28:269-75.

7 Brilla CG, Funck RC, RuppH. Lisinopril-mediated Brilla CG, Funck RC, RuppH. Lisinopril-mediated
regression of myocardial fibrosis in patients with hypertensive heart disease. Circulation 1999;100:I-362.
} 